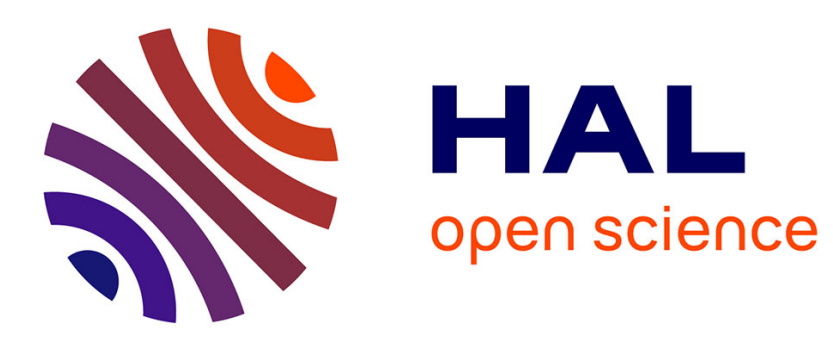

\title{
Minimization principles for the evolution of a soft sea bed interacting with a shallow
}

\author{
Afaf Bouharguane, Bijan Mohammadi
}

\section{To cite this version:}

Afaf Bouharguane, Bijan Mohammadi. Minimization principles for the evolution of a soft sea bed interacting with a shallow. International Journal of Computational Fluid Dynamics, 2012, 26 (3), pp.163-172. 10.1080/10618562.2012.669831 . hal-00789827

\section{HAL Id: hal-00789827 \\ https://hal.science/hal-00789827}

Submitted on 19 Feb 2013

HAL is a multi-disciplinary open access archive for the deposit and dissemination of scientific research documents, whether they are published or not. The documents may come from teaching and research institutions in France or abroad, or from public or private research centers.
L'archive ouverte pluridisciplinaire HAL, est destinée au dépôt et à la diffusion de documents scientifiques de niveau recherche, publiés ou non, émanant des établissements d'enseignement et de recherche français ou étrangers, des laboratoires publics ou privés. 


\title{
Minimization principles for the evolution of a
}

\section{soft sea bed interacting with a shallow sea}

\author{
Afaf Bouharguane \& Bijan Mohammadi \\ Institut de Mathématiques et de Modélisation de Montpellier \\ Université Montpellier II, CC51, 34095 Montpellier, France \\ Draft for IJCFD
}

\begin{abstract}
Minimization principles are used in fluid-structure coupling to model sandy sea beds evolution. The sandy bed is seen as a structure with low stiffness. We start with a model problem featuring the behavior of the coupling. Then water motion in shallow domains is described by the Saint Venant equations. This coupling bringing the bottom sea changes is based on the assumption that the bed adapts to the flow in order to minimize some energy quantity together with minimal sand transport. The approach is shown being equivalent to the use of an Exner equation for the bed with a nonlocal expression for the sediment transport flux. Because the choice of the functional permits to include different physical quantities one think to play a role in the bottom morphodynamics, the approach is also a tool for validating different modelling hypothesis.
\end{abstract}

Keywords: Sandy beach morphodynamics, shape optimization, fluidstructure coupling, Saint Venant Equations, sensitivity analysis, Exner equation. 


\section{Introduction}

Coastal morphodynamics occur over a broad range of time and length scales. Some events occur over small space and time scales such as the removal of sediment from the toe of coastal structures which often occurs and recovers completely during the course of a single tide (hourly basis). The next time and space scales concern storm response lasting for a few tides (say daily basis). Here the beach can be modified both long and cross-shore by hundreds of meters. Recovery between storms will take longer (weekly basis). This classification can continue with seasonal and inter-annual variability etc. In general, the spatial scale increases with the time scale and therefore long-shore transport gains importance over cross-shore with increasing time scale.

The literature on coastal morphodynamics is huge $[6,7,8]$ and concerns, for instance, dunes morphodynamics, sediment transports using fluid-induced shear in the sediment modelling, sea bed friction and the feedback of bed shapes on the flow, global beach morphodynamics based on long and cross-shore fluxes and beach lines morphodynamics.

This work is on the application of control theory to the evolution of sandy sea beds. We aim at proposing a formulation for sea bed evolution based on fluid and structure coupling using minimization principles. The time scales of interest are below a day and recoveries between storms or seasonal and interannual variabilities are out of scope. In the literature, the question of sea bed morphodynamics is, for instance, treated through the solution of an Exner equation with some adhoc flux expression [21]. The approach is shown to have a similar interpretation, but with a nonlocal flux term, as in some already existing models $[23,24]$.

In the past we used minimization principle to design defence structures against beach erosion $[3,4]$. In these works, the designed structures were independent of time and were built once for all. Here, we would like to go one step further giving the possibility to the structure to change in time. One particular 
case is then the sea bed is seen as a structure with low stiffness. The fundamental assumption is that the bed adapts to the flow by some sort of optimal sand transport in order to minimize some energy expression. Optimal transport is seen here as minimal change in the bed shape. The approach is not limited to the functionals we consider here, nor by the flow equations which can be more sophisticated.

The paper starts with the problem of sea bed motion formulated as an optimal control problem. A model problem is used to feature the different ingredients in the coupling procedure. Then a more sophisticated flow model in shallow water is introduced. Follows a discussion on the choice of cost functions. A comparison is then made between modelling the transport of sediments by the Exner equation and by our minimization principle. The paper also shows some simulations featuring qualitative behavior of the approach.

\section{A 1D model problem}

Let us illustrate our purpose on a model problem based on an analytical expression for water elevations linked as a function of incoming waves and the bottom sea expression. Consider a domain with a bed defined as a function $\psi(t, x): \mathbb{R}^{+} \times[-100 m,-20 m] \rightarrow \mathbb{R}^{+}$. We want to model the changes in this bed with time due to water elevations $h(t, x)$. Let us start with the following simple water elevation expression:

$$
h(t, x)=h_{0}+\alpha \sin \left(\omega x\left(h_{0}-\psi(t, x)\right)\right),
$$

where $h_{0}$ indicates the flow level at rest, $0 \leq \alpha<1$ and $\omega>0$. This is a monochromatic wave, but one could consider superimposed waves. This is an a priori and unphysical choice, made for illustration. Other analytical functions can be considered. We consider $h_{0}=2 \mathrm{~m}, \omega=0.5 \mathrm{~Hz}$ and $\alpha=0.1 \mathrm{~m}$. The bed is supposed initially flat $\psi(0, x)=1 \mathrm{~m}$. To close this model, we need to provide a model for $\psi(t, x)$. In this paper we suppose that $\psi$ will adapt to the flow 
elevation in order to damp some of its energy expressed through a functional $J(h(t, x, \psi(t, x)))$. The choice of $J$ is free at this point. Let us consider $J$ as the sum of the $L^{2}$ norm of $h$ in space and a term forcing the bottom to stay close to its original shape:

$J(h(t, x, \psi(t, x)))=\frac{1}{2} \int_{-100}^{-20}\left(h^{2}(t, x)+\beta(s(t, x)-s(0, x))^{2}\right) d x, \quad$ with $\beta \geq 0$.

The second term in the functional is to indicate that we assume the bottom adapting to the flow but also minimizing its own variations. We will discuss the underlying optimal transport issue in section 6 . An example of evolution model from time $t$ to time $t+\delta t$ for the flow and its bottom can be given by:

$$
\left\{\begin{array}{l}
\psi(t+\delta t, x)=\psi(t, x)-\Pi\left(\delta t \rho \nabla_{\psi} J(\psi(t, x), h(t, x))\right), \\
h(t, x)=h_{0}+\alpha \sin \left(\omega x\left(h_{0}-\psi(t+\delta, x)\right)\right) .
\end{array}\right.
$$

$\Pi$ is a projection operator and permits to account for possible presence of rigid parts in the bed (i.e. rocks) or a bed constituted with different type of sands permitting different local regularity for the bed shape. Here, we suppose the bed is homogeneous with only one type of sand and take $\Pi=I d . \rho \geq 0$ models the receptivity of the bed. We will see in section 8 how this can be linked to the porosity of the bed. Figure 1 shows snapshots of the evolutions of the bottom shapes and water elevations with 200 iterations of (2) with $\rho=0.002$ and $\delta t=3 s$ on a 500 points mesh. Based on the choice of $\omega$ the coupling takes place about four times every period. One can see that the bottom has adapted in order to reduce water elevations.

For this model problem the first optimality condition $\left(\nabla_{\psi} J=0\right)$ can be used to exhibit equilibrium solutions. It appears that, for $\beta=0$, because $h(t, x)>0$ and $x \neq 0$, the gradient vanishes where $\cos \left(\omega x\left(h_{0}-\psi(t, x)\right)\right)=0$ and therefore for

$$
\psi(t, x)=h_{0}-\frac{(2 k+1) \pi}{2 \omega x}, \quad \text { for } \quad k \in \mathbb{Z}
$$




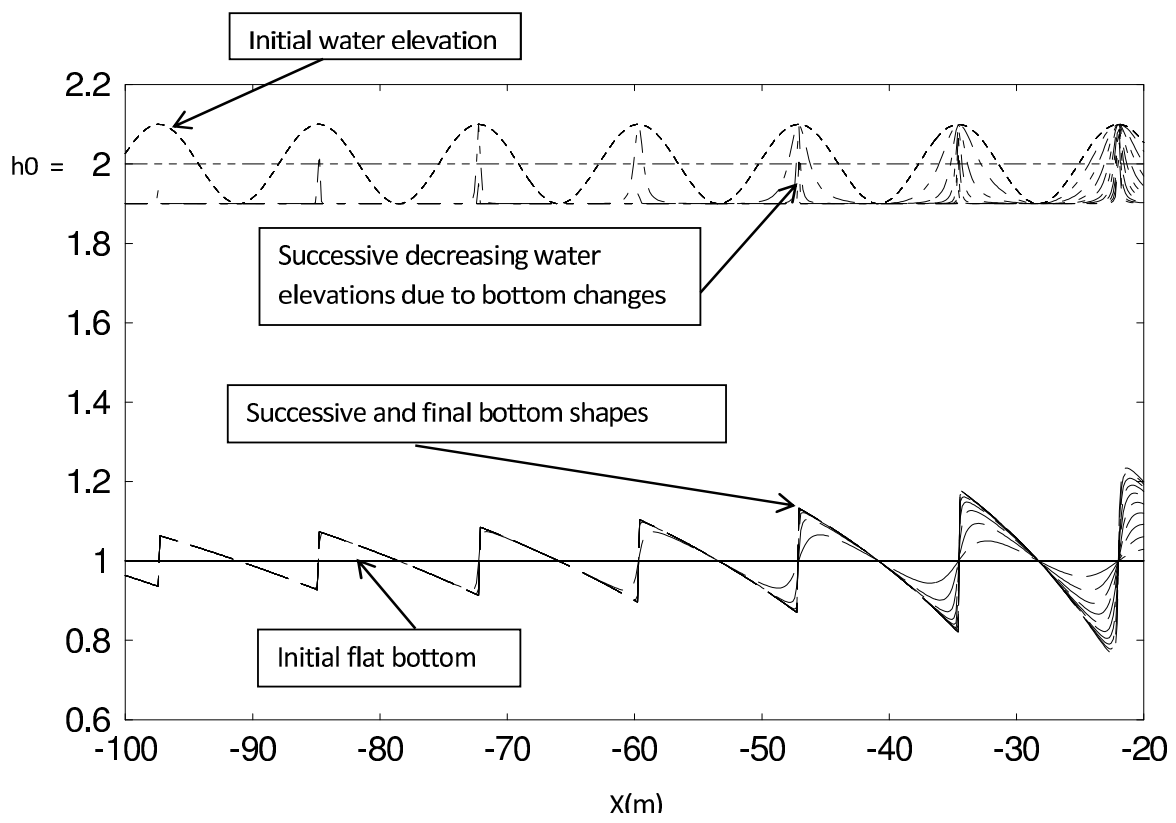

Figure 1: Coupling based on (2), starting from a flat bottom with $\beta=0$.

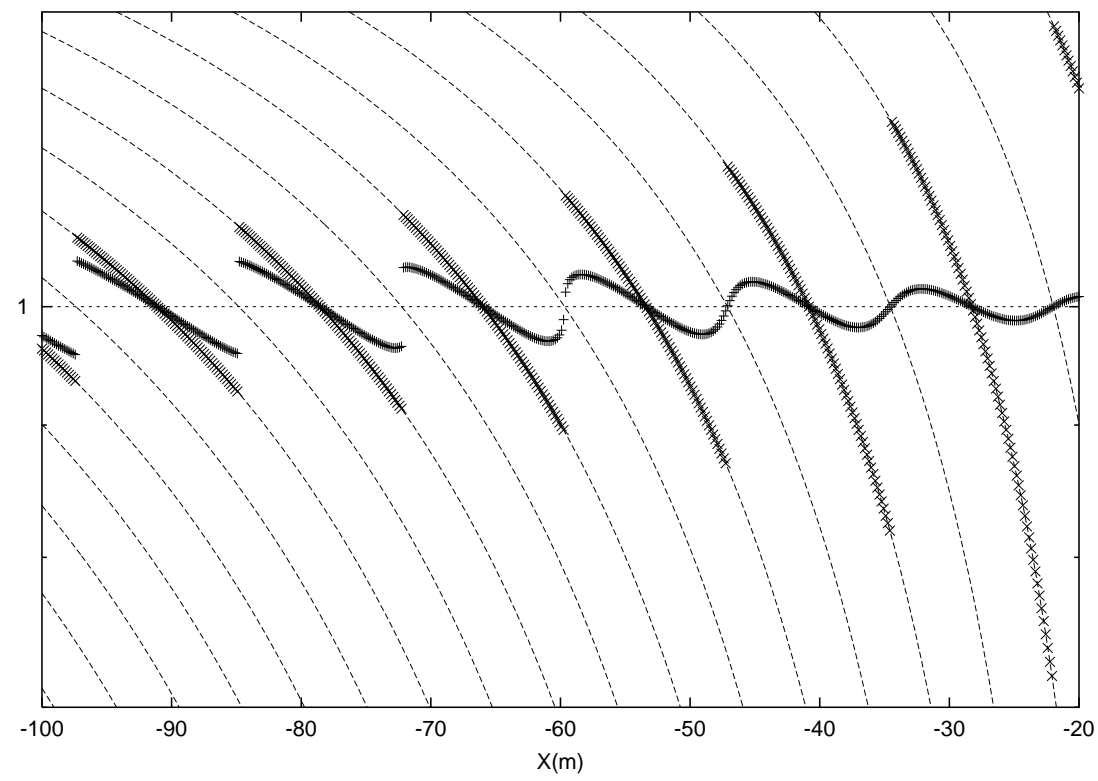

Figure 2: Regions where equilibrium solution exists (for $\beta=0$ ) from the first optimality condition and the equilibrium bottom shapes found by the gradientbased coupling for functional (1) with $\beta=0(\mathrm{x})$ and $\beta=100(+)$. 

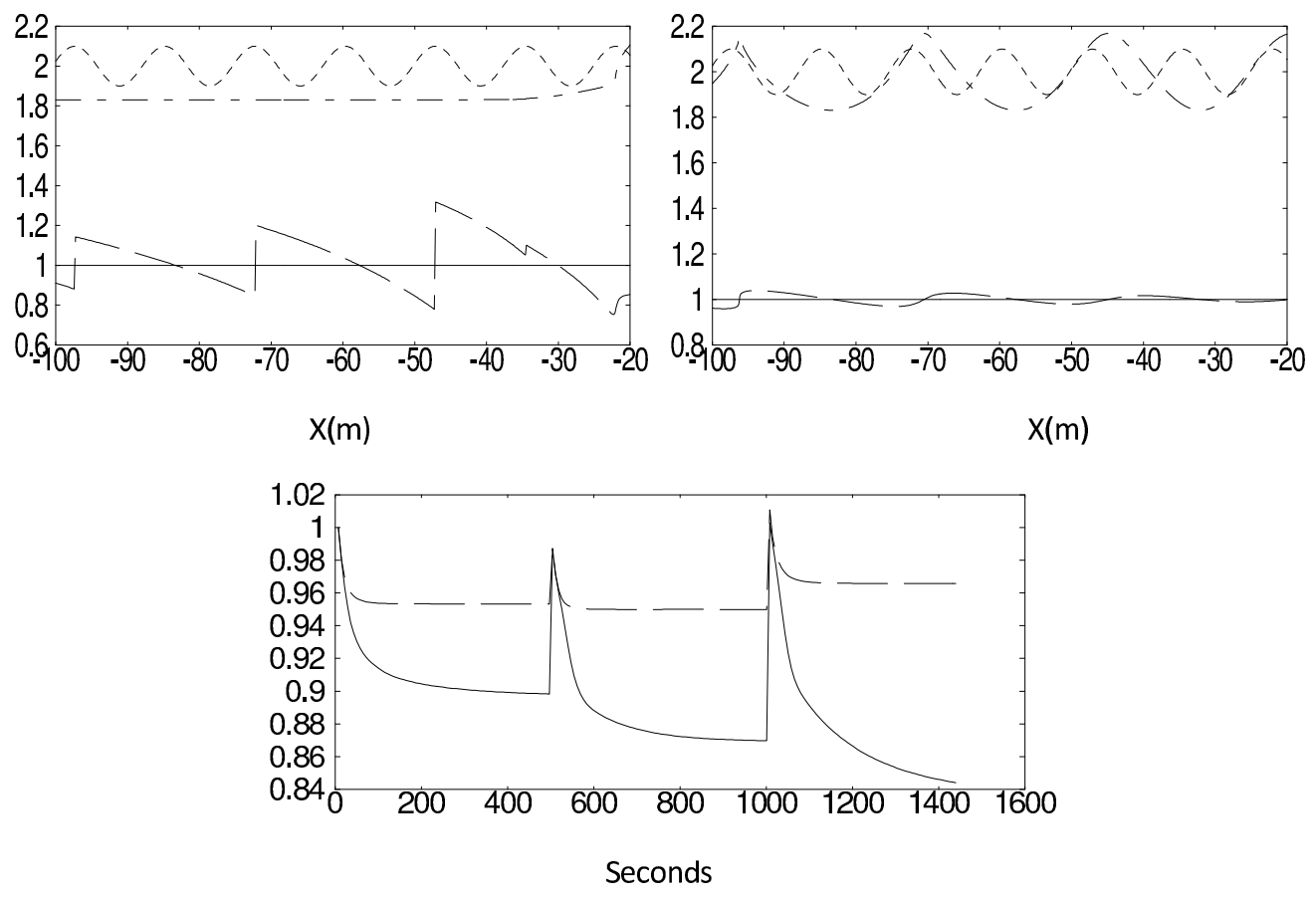

Figure 3: Upper: Initial and final water elevations after the bed has adapted to three water condition changes for $\beta=0$ (left) and $\beta=100$ (right). Lower: normalized histories of $\int_{-100}^{-20} h^{2} d x$ for $\beta=0$ and $\beta=100$ (dashed) during coupling iterations. Under constraint the water elevation control is less efficient. The couple bed-flow reaches an equilibrium until a change in the flow conditions occurs. 
These orbits are shown in Figure 2 and compared with the solutions by the coupling in the case of $\beta=0$ and $\beta=100$. There are two possible equilibrium solutions corresponding to even and odd $k$ 's. The coupling solutions is along with odd $k$ 's. This is an example of non-uniqueness. The gradient method appears to pick the solution which shows a compromise between variation and regularity. Indeed, passing by all orbits would have reduced the bottom variations but the outcome would have been less regular.

Figure 3 shows the evolution of the $L^{2}$ norm of water elevations for $\beta=0$ and $\beta=100$ with two water conditions changes during the coupling where we respectively applied $(\omega=0.35 \mathrm{~Hz}, \alpha=0.13 \mathrm{~m})$ and $(\omega=0.24 \mathrm{~Hz}, \alpha=0.17 \mathrm{~m})$. This is exactly what is observed in nature: the bed moves until the couple bedflow reaches an equilibrium. This stable configuration is broken by a change in the flow conditions (i.e. incoming waves directions and intensities).

As we will see in section 8 , several morphodynamic models in literature are in a divergence form:

$$
\psi_{t}+\frac{1}{1-\lambda_{p}(x)} \operatorname{div}(q(x, t))=0, \quad \psi(t=0, x)=\text { given }
$$

where $\lambda_{p}(x) \in[0,1[$ is the porosity of the bed. This can be linked to the present minimization formulation giving a systematic approach for the derivation of the flux term:

$$
\psi_{t}=-\frac{1}{1-\lambda_{p}(x)} q_{x}=-\rho J_{\psi}
$$

and integrating, one has:

$$
q(x, t)-q(-100, t)=\int_{-100}^{x} \rho(s)\left(1-\lambda_{p}(s)\right) J_{\psi}(s, t) d s,
$$

where one has supposed that both $\rho$ and $\lambda_{p}$ depend on the space (i.e. non homogeneous bed). Hence, to have $q$ one needs $q(-100, t)$ which is one extra information required to close the model in comparison to the minimization approach. And this data is usually difficult to obtain as it requires bed mea- 
surements. On the other hand, the flux approach can be reformulated as a minimization problem finding the related cost function.
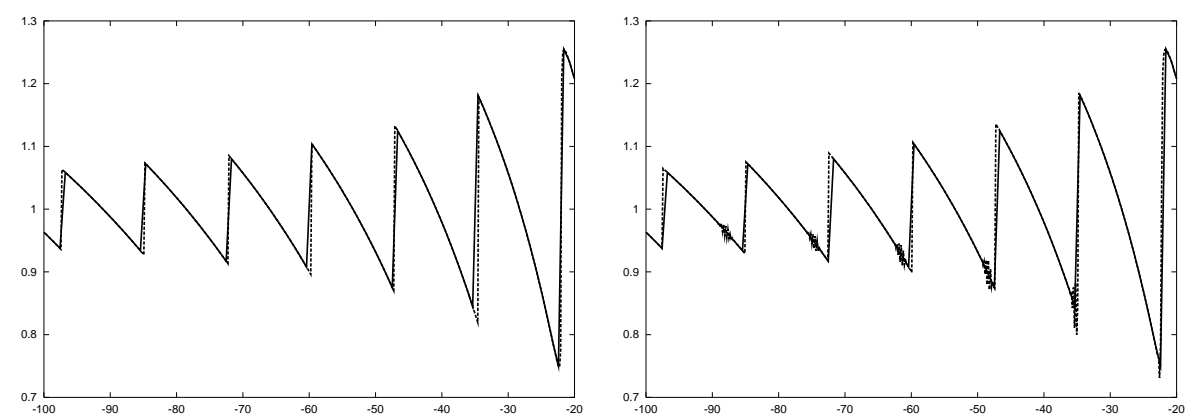

Figure 4: Final bottom shapes with the minimization approach and with the solution of the associated divergence form, using a first order upwind discretization for the space derivative (left) and with second order central differences (right).

Now, let us consider a numerical implementation of (3) with constant $\rho$ and $\lambda_{p}$. To solve this PDE, one needs to provide boundary conditions for $\psi$ which are again extra informations not necessary in the minimization approach. Figure 4 shows a comparison of the bottom shapes with the above minimization based coupling and with the associated divergence form with boundary conditions $\psi_{x x}(-100)=\psi_{x x}(-20)=0$. The results show the need for upwinding. We will see in section 7 how to evaluate the gradient of the functionals by an adjoint method. The previous comparison indicates that the gradient contains implicitly the information on the boundary conditions on the bed required if one uses fluxbased morphodynamics models.

The approach we described through this simple model problem can be applied to more complicated situations involving realistic flow models. The choice of the functional permits to include different physical quantities one would think to play a role in the bottom morphodynamics. The approach is therefore also a tool for validating different modelling hypothesis. This will be discussed in section 6 . 


\section{Interactions between the flow and its bed}

Now, one would like to go one step further considering two dimensional situations with a realistic flow model in shallow domains. The sea bed $\psi: \Omega \subset$ $\mathbb{R}^{2} \rightarrow \mathbb{R}^{+}$changes with time following the changes in the state given by the flow conditions U. $\psi$ is a univocal function with some regularity. We use a bed parameterization based on the bathymetry given at all the nodes of the fluid mesh. This is called a CAD-free parameterization [9].

The model for the sea bed evolution is based on the minimization of a time dependent functional $J(\psi, \mathcal{U}(\psi))$ where $\mathcal{U}(\psi)=\{\mathbf{U}(\psi, \tau, x, y), \tau \in[t-$ $T, t],(x, y) \in \Omega\}$ gathers the state evolution in time, solution of a state equation. We discuss the choice of functionals in section 6 .

To make the physical model more realistic, we consider the shallow water equations as state equations for the fluid with $\mathbf{U}={ }^{t}(h, h \mathbf{u})$ where $\mathbf{u}={ }^{t}(u, v)$ is the depth-averaged velocity with $u$ and $v$ the scalar components in the horizontal directions and $h$ the local water depth:

$$
\mathbf{U}_{t}+F(\mathbf{U}, \psi)=0, \quad \text { with initial and boundary conditions, }
$$

where

$$
F(\mathbf{U}, \psi)=\left(\begin{array}{c}
\nabla \cdot(h \mathbf{u}) \\
\nabla \cdot(h \mathbf{u} \otimes \mathbf{u})+g h \nabla(h+\psi)
\end{array}\right)
$$

As in our model problem, minimization of $J$ can be seen as solution of

$$
\left\{\begin{array}{l}
\psi_{t}=-\rho \nabla_{\psi} J(\psi, \mathcal{U}(\psi)) \\
\psi(t=0)=\text { given }
\end{array}\right.
$$

where $\rho$ is a positive parameter which depends on the porosity of the bed (see section 8). The physical time scales for the fluid and structure (bed motion) are quite different. Indeed, flows have time scales of the order of seconds and the 
sea bed motion takes place over hours.

The cost function involves this state evolution as, for instance, in:

$$
J(\psi, \mathcal{U}(\psi))=\int_{t-T}^{t} j(\psi, \mathbf{U}(\psi, \tau)) d \tau
$$

$T$ indicates a time dependency window and also permits to introduce a difference in time scales between sea bed and flow motions. We discuss its choice in section 6.

\section{Flow solver}

Shallow water equations are discretized by a finite volume formulation [2]. Our finite volume implementation preserves steady state solutions on non flat sea beds in the absence of perturbations [14, 15]. It is also suitable to capture wetting and drying phenomena $[17,16]$. Four boundary conditions are needed at slip, inlet, shoreline and outlet boundaries. The slip boundary condition (u.n = 0 ) is naturally taken into account in a finite volume formulation. The outlet condition is a transmissive boundary condition. Values at boundary cells are obtained by second order extrapolations normal to the boundary from the values inside the domain. To describe incoming waves we use an absorbing/generating inlet boundary condition where the values of water depth are prescribed. In subcritical regimes, characteristic curves and Riemann invariants provide normal velocity. In open sea we allow for reflected waves to freely exit the domain [18].

\section{Simulations details}

To simulate sea conditions, the water wave elevation at the inlet is represented by the addition of $N$ monochromatic waves:

$$
h(t)=h_{0}+2 A \sum_{i=1, . ., N-1} \sin \left(\frac{\omega_{i}+\omega_{i+1}}{2} t\right) \cos \left(\frac{\omega_{i}-\omega_{i+1}}{2} t\right) .
$$


$A$ is the water wave amplitude at inlet, $h_{0}$ the water height at rest and $\omega_{i}=$ $2 \pi / T_{i}$ are wave pulsations. With five modes, typical values of periods $T_{i}$ we consider are $3.5,3,2.3,2,1.5$ seconds. We consider the case of an experiment conducted at the Sogreah 3D wave basin in Grenoble (France) of side $30 \mathrm{~m}$ with $A=0.12 \mathrm{~m}$ and $h_{0}=0.765 \mathrm{~m}$. More realistic conditions can be applied following a Jonswap distribution [20, 19].

Time integration is explicit for the flow. Due to what we said on the difference of time scales between fluid motion and changes in sea beds, several time steps will be taken (typically 100) in the flow solver before a new time step by the bed model described by the minimization iterations.

\section{Cost function}

One of the main ingredients in the chain of control is the cost function to be minimized. One expects the bed to act as a flexible structure and to adapt to flow conditions in order to minimize some energy-based functional. One can, for instance, consider an energy made of water mechanical energy and involving a constraint on sand displacements requiring minimal topobathymetry changes from the bathymetry $\psi(t-T)$ at the beginning of a time interval of influence $[t-T, t]:$

$$
J_{1}(\psi)=\int_{t-T}^{t} \int_{\Omega}\left(\frac{1}{2} \rho_{w} g \eta^{2}+\rho_{s} g(\psi(\tau)-\psi(t-T))^{2}\right) d \tau d \Omega
$$

where $\Omega$ is the physical domain, $\rho_{w}$ and $\rho_{s}$ are respectively the water and sand density and $\eta$ is the deviation of the wave elevation from a low frequency component evaluated using a time interval $T$. This is a function of space and time and also depends on the bathymetry:

$$
\eta(x, y, \psi, t)=h(x, y, \psi, t)-\frac{1}{T} \int_{t-T}^{t} h(x, y, \psi, \tau) d \tau .
$$


This functional aims at reducing both wave elevations and changes in the bed shape.

In functional (6), one could have considered water wave amplitude $A$ instead of elevations:

$$
A(x, y, \psi)=\max _{t \in[t-T, t]} \eta(x, y, \psi, t)
$$

and

$$
\tilde{J}_{1}(\psi)=\int_{\Omega} \frac{1}{2} \rho_{w} g A^{2} d \Omega+\int_{t-T}^{t} \int_{\Omega} \rho_{s} g(\psi(\tau)-\psi(t-T))^{2} d \tau d \Omega
$$

$J_{1}$ and $\tilde{J}_{1}$ are equivalent on compact domains and a reduction of $J_{1}$ leads to the same for $\tilde{J}_{1}$. From an optimization point of view, one prefers $J_{1}$ because it is differentiable. In all cases, we have observed that controlling water wave energy is crucial to fight erosion. As, this is responsible for sediments put in suspension by generating orbital velocity $[8,7,4]$.

A fundamental idea motivating the introduction of the second term in the functional (6) is the concept of optimal transport [10,11, 12]. In other words, one thinks that the bed will adapt to the flow in some optimal way in term of sand transport.

We assume that the influence of water conditions on a sandy bed at a given instant does not involve time history of more that a few wave periods. $T$ is a parameter to be assimilated using experimental data. Its choice is important as shown in Figure 5. It illustrates bottom seas after six hours with the functional (6) for two different values of the interval of influence $T=5$ and $T=10$ seconds starting from a linear initial profile for the bed. The picture is along the crossshore centerline of the domain. The long-shore deviations from these profiles are negligible. It appears that larger $T$ produce less sand motion. This is reasonable because when $T$ is large deformations will be based on more flow features. This is similar then to a multi-point optimization with more and more constraints. And increasing the number of constraints in general restraint the admissible deformation. 


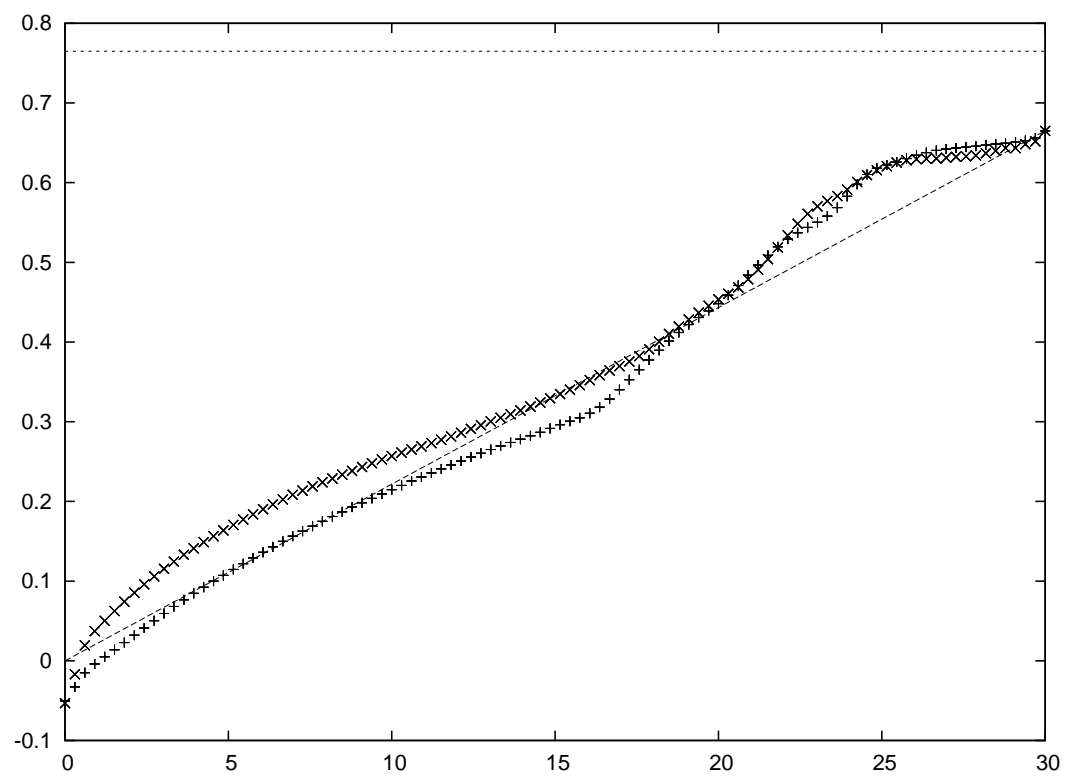

Figure 5: Starting from a linear bed, bathymetries along the cross-shore centerline of the domain after six hours for the functional (6) for $T=5 s(+)$ and $T=10 s(\mathrm{x})$. Dashed lines are initial bathymetry and water level at rest.

The previous functional involves water elevations. In the same way, one could imagine that sea bed will adapt to the flow in order to reduce its horizontal velocity gradients with minimum variation in bathymetry. A second functional can then be built:

$$
J_{2}(\psi)=\int_{t-T}^{t} \int_{\Omega}\left(\left\|\nabla_{x y} \mathbf{u}\right\|+\rho_{s} g(\psi(\tau)-\psi(t-T))^{2}\right) d \tau d \Omega,
$$

where $\nabla_{x y}$ indicates the spatial gradient and the norm stands for $\left\|\nabla_{x y} \mathbf{u}\right\|=$ $\left(u_{x}^{2}+v_{x}^{2}+u_{y}^{2}+v_{y}^{2}\right)^{1 / 2}$.

There is an extra constraint to account in cases we assume the domain is closed and the amount of available sand given:

$$
\int_{\Omega} \psi(t, x, y) d \Omega=\int_{\Omega} \psi(0, x, y) d \Omega
$$

Here, we assume open sea conditions where extra sand can be brought in by the 
flow if predicted so. Then, the constraint on $\psi$ remaining as close as possible to the initial bathymetry is absolutely necessary in order for the algorithm not to remove all water, hence vanishing the functional.

Figure 6 shows an example of sea bed changes along the cross-shore centerline of the domain due to interactions with water motion based on the minimization of (6) and (8) after about six hours. The approach predicts the apparition of either two or three longshore sand bars. Water elevation in time is prescribed on the left boundary which creates waves entering normal to this boundary. The starting bed profile is linear. Functional (6) especially predicts beach nourishment.

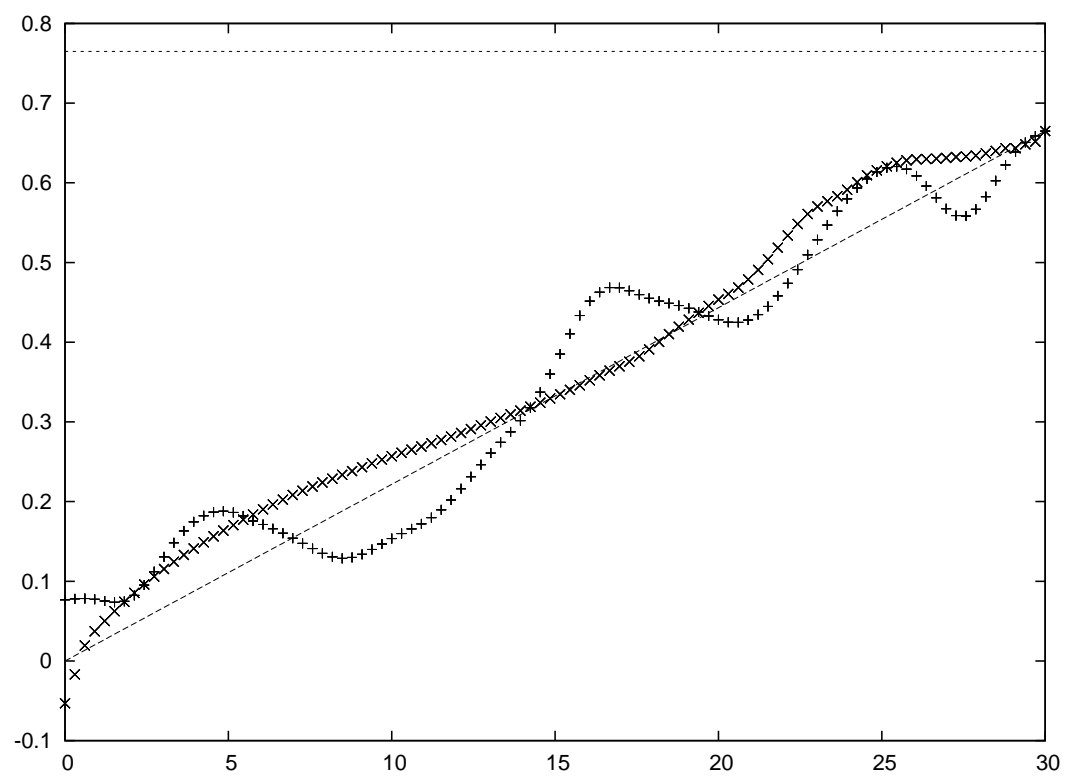

Figure 6: Starting from a linear bed, final bathymetries for a functionals (6) (x) and $(8)(+)$ for the same sea bed and for $T=5 s$ along the cross-shore centerline. Dashed lines are initial bathymetry and water level at rest.

Figure 7 shows the evolution of two different initial bathymetries along the cross-shore centerline: a linear and storm profiles. We can see that a initial sea bed is quite determinant for the evolution of the bed shape and the equilibrium profile achieved. The bed shape has however the same composition with two bars. 


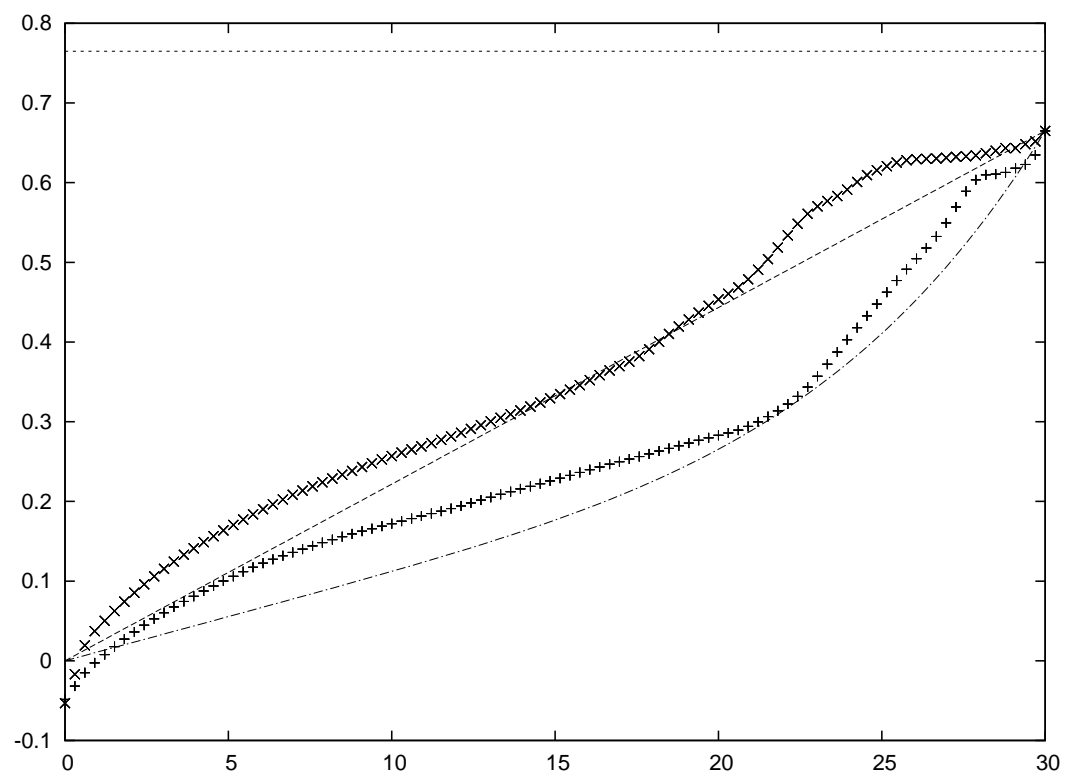

Figure 7: Starting from two different bed shapes, final bathymetries for functional (6) for $T=5 \mathrm{~s}$ along the cross-shore centerline.

To give an indication on the long-shore deviation and also the history of the coupling, Figure 8 shows the final bed and the evolution in time of the functional (6) over six hours starting from a linear bed and then from a bed with local addition of a large amount of sand. The final bed does not see this perturbation. The flow conditions drive the coupling. In other words, one cannot expect much differences in the outcome if a sandy bottom is locally modified with the addition of removable ingredients. We have experienced that adding immersed geotubes can on the other hand help rebuilding a beach which is otherwise under erosion $[4,5]$.

One sees some equilibrium appearing after about two hours around which the bed then fluctuates.

\section{Sensitivity evaluation}

Sensitivity evaluation in large dimension needs an adjoint variable approach to make the cost of the evaluation independent from the size of the control 

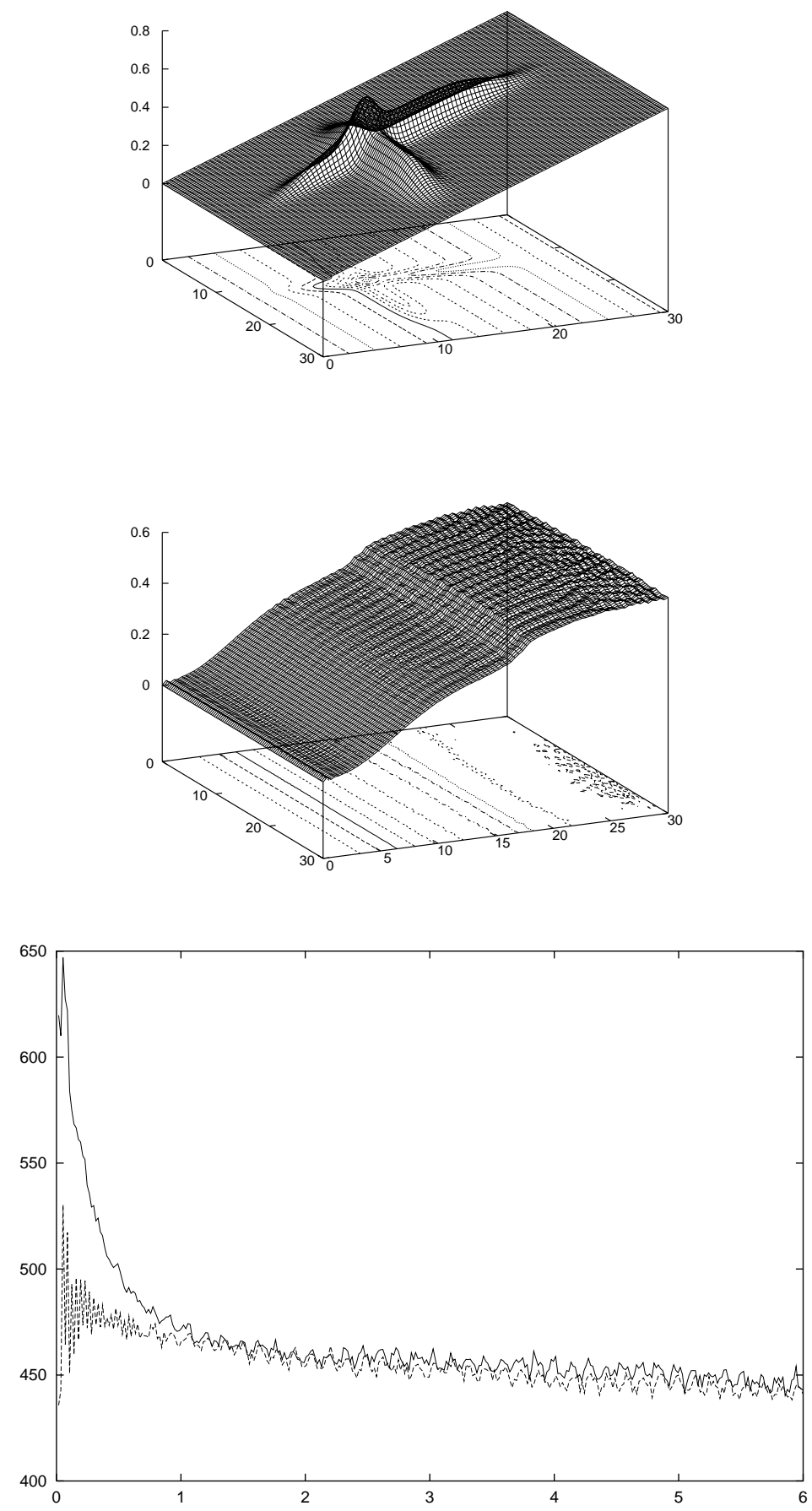

Figure 8: Upper: same final bed (middle picture) is found starting either from a linear or a more complex bed (top picture). Lower: evolution of the functional (6) over six hours for the two initializations. 
space. In time dependent problems, this implies storage of all intermediate states which can be optimized by check-pointing technics [28]. In this section, we briefly describe the adjoint method with a time dependent state equation (the Saint Venant equations in our case). This will be implemented by automatic differentiation $[27,9,13]$. Let us consider the following formal dependency chain:

$$
\psi \rightarrow\{\mathbf{U}(\psi, \tau), \tau \in[0, T]\} \rightarrow J(\psi, T)
$$

Here, $\psi$ is the independent variable and $\mathbf{U}$ and $J$ are the dependent ones. $\mathbf{U}(\psi, \tau)$ are solutions in time of

$$
\mathbf{U}_{t}+F(\mathbf{U}, \psi)=0, \quad \mathbf{U}(0)=\mathbf{U}_{0}(\psi) .
$$

To be accurate, one should have also to consider the independent physical parameters such as those describing the waves. But, this would have introduced unnecessary complications into notations.

Now consider a functional involving an integral over time like those we are interested in:

$$
J(\psi, T)=\int_{(0, T)} j(\psi, \mathbf{U}(\psi, t)) .
$$

Linearizing $J$ one has:

$$
J_{\psi}(\psi, T)=\int_{(0, T)}\left(j_{\psi}+j_{\mathbf{U}} \mathbf{U}_{\psi}\right)
$$

In this expression only $\mathbf{U}_{\psi}$ is costly to get as it requires the linearization of the shallow water equations.

The linearized state equation:

$$
\left(\mathbf{U}_{\psi}\right)_{t}+F_{\psi}(\mathbf{U}, \psi)+F_{\mathbf{U}}(\mathbf{U}, \psi) \mathbf{U}_{\psi}=0, \quad \mathbf{U}_{\psi}(0)=\mathbf{U}_{0}^{\prime}(\psi),
$$


permits to write for all function $\mathbf{V}$ (where $\mathbf{V}$ has the same structure than $\mathbf{U}$ ):

$$
0=\int_{(0, T) \times \Omega}\left(\left(\mathbf{U}_{\psi}\right)_{t}+F_{\psi}(\mathbf{U}, \psi)+F_{\mathbf{U}}(\mathbf{U}, \psi) \mathbf{U}_{\psi}\right) \mathbf{V} .
$$

Introducing the adjoint operator $F_{\mathrm{U}}^{*}$, it gives:

$$
0=\int_{(0, T) \times \Omega}\left(-\mathbf{V}_{t}+F_{\mathbf{U}}^{*}(\mathbf{U}, \psi) \mathbf{V}\right) \mathbf{U}_{\psi}+\int_{\Omega}\left[\mathbf{V} \mathbf{U}_{\psi}\right]_{0}^{T}+\int_{(0, T) \times \Omega} \mathbf{V} F_{\psi}(\mathbf{U}, \psi) .
$$

Let us introduce a backward adjoint problem:

$$
\mathbf{V}_{t}+F_{\mathbf{U}}^{*}(\mathbf{U}, \psi)=j_{\mathbf{U}}, \quad \mathbf{V}(T)=0
$$

Therefore, with $\mathbf{V}$ solution of the backward adjoint equation (11) with the chosen final condition one has:

$$
\int_{(0, T) \times \Omega} j_{\mathbf{U}} \mathbf{U}_{\psi}=\int_{\Omega} \mathbf{V}(0) \mathbf{U}_{0}^{\prime}(\psi)-\int_{(0, T) \times \Omega} \mathbf{V} F_{\psi}(\mathbf{U}, \psi) .
$$

If there is no direct dependency between the initial condition $\mathbf{U}(0)$ and $\psi$ the first term in the right-hand-side vanishes. Also, for the Saint Venant equations the direct dependency in $\psi$ is in $g h \nabla \psi$ in the equations for $h \mathbf{u}$, see (4). Denoting $\mathbf{V}=\left(v_{1}, \mathbf{v}_{\mathbf{2}}\right)^{t}$ with $\mathbf{v}_{\mathbf{2}}$ the adjoint variable associated to $\mathbf{u}$, one has in weak form:

$$
\int_{(0, T) \times \Omega} \mathbf{V} F_{\psi}(\mathbf{U}, \psi)=-\int_{(0, T) \times \Omega} g \nabla \cdot\left(h \mathbf{v}_{\mathbf{2}}\right) .
$$

We have used either slip or Dirichlet boundary conditions for the velocity which give for the corresponding adjoint variable slip or homogeneous Dirichlet conditions removing the boundary term in weak form. An important point here is that, unlike with the linearized equation, with $\psi$ of any dimension $\mathbf{V}$ is computed only once. One remarks however that states $\mathbf{U}$ are needed in reverse order because the backward integration in (11). This can be further simplified in some special cases where the functional enters the admissibility domain of incomplete sensitivity concept [9]. 


\section{Minimization principle and the Exner equation}

Let us reconsider the following equation which in discrete form minimizes $J(\psi)$ :

$$
\psi_{t}=-\rho \nabla_{\psi} J, \quad \psi(t=0, x, y)=\psi_{0} .
$$

where $\rho$ characterizes the ability of sand to be put in motion by water. The parameter $\rho$ and the interval of influence $T$ are the two physical data of the bed model. They are obtained from observation site for a given site.

One well-known approach to model sea bed motion is through the Exner equation [21]. This equation models the conservation of mass between the bed and transported sediments.

$$
\psi_{t}+\frac{1}{1-\lambda_{p}} \nabla \cdot q=0, \quad \psi(t=0, x, y)=\text { given }
$$

where $\lambda_{p} \in[0,1[$ is the porosity of the bed and $q$ is the sediment transport flux. To estimate this flux $q$, scientists propose a variety of formula such as the Meyer-Peter \& Müller or Gras formulas [25]. The former suggests that the flux $q$ is directly related to the shear stress and the latter to the fluid velocity .

One remarks then that $1 /\left(1-\lambda_{p}\right)$ plays the role of $\rho$ in the minimization equation (12) and as expected $\rho$ increases with the porosity of the bed. Indeed, one expects the influence of a same flow condition on the bed to increase with the bed's porosity. Let us suppose that the porosity is constant everywhere, then the analogy suggests that

$$
\nabla \cdot q=\nabla_{\psi} J
$$

Consider a one dimensional situation. One sees that the previous expression 
suggests a nonlocal definition for $q$ :

$$
q(t, x)=q(t,-\infty)+\int_{-\infty}^{x} \nabla_{\psi} J(\zeta) d \zeta
$$

where without harm one can suppose $q(t,-\infty)=0$ and $\nabla_{\psi} J(t, \zeta) \rightarrow 0$ when $x \rightarrow-\infty$ as the influence of the flow on the bed decreases offshore with the flow depth increasing. This indicates that, compared to the minimization formulation, these approaches need be applied on a larger domain in order to reduce the uncertainties one might have on $q(t,-\infty)$. The nonlocal term depends on the choice of the cost function. Also, as saw in the model problem in section 2, one needs to provide boundary conditions for $\psi$ for the solution of the transport equation.

Let us analyze what the approach gives for simple functionals in one dimension in space with $J=\frac{1}{2} u_{x}^{2}$. Minimizing $J$ would force the bottom to adapt in order to reduce gradients in the flow. This is similar to what we expect minimizing (8). For this functional $q$ reads:

$$
q(t, x)=\int_{-\infty}^{x}\left(1-\lambda_{p}\right)^{1-n} u_{\zeta} u_{\psi \zeta} d \zeta
$$

and integrating by part, we get

$$
q(t, x)=-\int_{-\infty}^{x}\left(\left(1-\lambda_{p}\right)^{1-n} u_{\zeta}\right)_{\zeta} u_{\psi} d \zeta+\left(1-\lambda_{p}(x)\right)^{1-n} u_{x}(t, x) u_{\psi}(t, x)
$$

This shows a local term plus a global correction involving second order derivatives of the state upstream. Similar nonlocal terms can be found, for instance, in a Fowler model for the motion of sand dunes sheared by a fluid flow [23].

\section{Choice of the functional}

To end the paper, let us discuss a few possible approaches for the choice of the functional in our approach. As in shape optimization problems, the choice of the 
functional depends on what is aimed for the outcome of the design. We assumed here that the target is a reduction of the energy of the wave with minimal sand transport. Based on this a priori assumption, we discussed in section 6 several choices, such as (6), (7) and (8).

Then in section 8 we linked our approach with the Exner equation. This gives another way to derive a functional from the Exner equation, under the mentionned hypothesis, using equality (14). This is something we would like to investigate in the future, but rather from a theoretical point of view. Indeed, the Exner equation being mainly used in one dimension in space, the extension to higher space dimension will again require a priori modelling hypothesis as in the previous approach.

Another interesting and powerful procedure would be data assimilation of available observations of historical erosion data basis. For instance, information on shoreline or bathymetry evolutions for a given beach can be accounted for in functional (6):

$J_{3}(\psi)=\int_{t-T}^{t} \int_{\Omega}\left(\frac{1}{2} \rho_{w} g \eta^{2}+\rho_{s} g(\psi(\tau)-\psi(t-T))^{2}\right) d \tau d \Omega+\int_{Q}\left(\psi-\psi_{o b s}\right)^{2} d q$,

where $Q(\subset] 0, t[\times \Omega)$ denotes the space-time domain, which is necessary limited, where observation data $s_{o b s}$ are available.

An alternative approach would be to account for observations through the minimization of a second functional. Bathymetry observations can be used as target to see which functional minimization best reproduces the history. For this we need to first parameterize our functional as, for instance, in the following choice:

$$
J_{4}(\psi)=\int_{t-T}^{t} \int_{\Omega}\left(\frac{1}{2} \rho_{w} g \eta^{2}+\gamma_{1}\left\|\nabla_{x y} \mathbf{u}\right\|+\gamma_{2} \rho_{s} g(\psi(\tau)-\psi(t-T))^{2}\right) d \tau d \Omega
$$


where $\gamma_{1}$ and $\gamma_{2}$ are positive constants to be found minimizing:

$$
D\left(\gamma_{1}, \gamma_{2}\right)=\int_{Q}\left(\psi\left(\gamma_{1}, \gamma_{2}\right)-\psi_{o b s}\right)^{2} d q
$$

Hence, eventually, we have two nested minimization problems. Data assimilation minimizing the error between predictions and measurements and having as outcome the coefficients in the functional we use in our minimization-based bathymetry evolution.

\section{Concluding remarks}

Minimization principles have been used to describe the coupling of a fluid in a shallow domain with a flexible and deforming structure. The coupling aims at reproducing a sandy sea bed evolution due to water motion. This study shows that the outcome of the coupling has suitable qualitative behavior predicting apparition of observed natural bars in sandy beaches. Several assumptions have been analyzed and the corresponding cost functionals described. Sensitivity analysis is used to minimize these functionals. Minimization iterations give the sea bed morphodynamics law.

The flow motion is described by the shallow water equations but this can be extended to more sophisticated models including dispersion effects. Also, the approach can obviously be applied to situations where the flow is described by a spectral approach and where time has been removed from the equations using separation of variables in time and space $[26,3,4]$.

It has been shown that the minimization approach is equivalent to solving an Exner equation for the bed with an original flux term. Our current research aims at linking this approach with other available models in the literature for bed transformation involving nonlocal fluxes [23].

The next step in the modelling is to go beyond qualitative validation by assimilating observation data. The two parameters of the model which need identification are the bed porosity and local time interval of influence between 
the flow and the bed. It is shown that the approach requires less informations than morphodynamics flux-based models where boundary conditions are necessary for the solution of the sediment transport equation.

This study also takes place in the context of shape optimization for unsteady flows where both time dependent and independent shapes can be targeted. Bottom sea motion is an example of the former and building defence structures against erosion [4] the latter.

\section{Acknowledgments}

The author would like to thank P. Azerad, F. Bouchette and F. Marche for helpful discussions around this work, especially in the definition of the functionals and the simulation conditions for the basin. This work is part of ANR project Mathocean (ANR-08-BLAN-0301-01).

The adjoint Saint Venant solver has been obtained using Tapenade AD tool developed at INRIA-Sophia Antipolis by L. Hascoet and his team Tropics http://www-sop.inria.fr/tropics/tapenade.html.

\section{References}

[1] Barré de Saint Venant A. J.-C. ( 1871). Théorie du mouvement nonpermanent des eaux, avec application aux crues des rivières et à l'introduction des marèes dans leur lit. C.R. Acad. Sc. Paris; Vol. 73, 147154.

[2] Eymard, R. Gallouët, T. Herbin, R. (2000). Finite volume methods. In Handbook of numerical analysis, vol VII, Ciarlet PG, Lions JL, editors. North Holland: Amsterdam, 729-1020.

[3] Azerad, P. Bouchette, F. Isebe, D. Mohammadi, B. (2008). Optimal shape design of coastal structures minimizing short waves impact. Coastal Engineering. Vol. 55/1. 
[4] Azerad, P. Bouchette, F. Isebe, D. Mohammadi, B. (2008). Shape optimization of geotextile tubes for sandy beach protection. International Journal for Numerical Methods in Engineering, Vol. 74, 1262-1277.

[5] Bouharguane A., Azerad P., Bouchette F., Marche F. and Mohammadi B., Low complexity shape optimization and a posteriori high fidelity validation, Disc. Cont. Dyn. Syst., Ser. B, 13, pp. 759-772, 2010.

[6] Dean, R.G. Dalrymple, R.A. (2004). Coastal processes with Engineering Applications, Cambridge University Press.

[7] Dean, R.G. Dalrymple, R.A. (1991). Water Wave Mechanics for Engineers and Scientists, World Scientific Publishing.

[8] Mei, C.C. (1989). The applied dynamics of ocean surface waves. World Scientific Publishing.

[9] Mohammadi, B. Pironneau, O. (2009). Applied shape optimization for fluids, $2^{\text {nd }}$ edition. Oxford Univ. Press.

[10] Vilani, C. (2003), Topics in optimal transportation, Graduate Studies in Mathematics series, AMS. 58.

[11] Brenier, Y. Benamou, J.D. (2000). A computational fluid mechanics solution to the Monge-Kantarovich mass transfer problem, Numerish Math. 84-3. 375-393.

[12] Mohammadi, B. (2007). Optimal transport, shape optimization and global minimization, C. R. Acad. Sci. Paris, 3511.

[13] Mohammadi, B. Pironneau, O. (2004) Shape Optimization in Fluid Mechanics, Annual Revue of Fluid Mechanics, 36-1.

[14] Audusse, E. Bouchut, F. Bristeau, MO. Klein, R. Perthame, B. (2004). A fast and stable well-balanced scheme with hydrostatic reconstruction for shallow water flows. SIAM J.Sci.Comp. 25/6, 2050-2065. 
[15] Audusse, E. Bristeau, M.-O. (2005). A well-balanced positivity preserving second order scheme for shallow water flows on unstructured meshes, J. Comp. Phys. Vol. 206, 311-333.

[16] Marche, F. Bonneton, P. (2006). A simple and efficient well-balanced scheme for 2D bore propagation and run-up over a sloping beach. in Proceedings of Int. Conf. Coast. Eng. ICCE, Springer.

[17] Marche, F. Bonneton, P. Fabrie, P. Seguin, N.A. (2002). Evaluation of well-ballanced bore-capturing schemes for 2D wetting and drying processes, IJNMF.

[18] Kobayashi. N. Desilva, GS. Watson, KD. (1989). Wave transformation and swash oscillation on gentle and steep slopes. J. Geophys. Res. Vol. 94, 951966 .

[19] Hasselmann, D.E. Dunckel, M. Ewing, J.A. (1980). Directional Wave Spectra Observed during JONSWAP 1973, Journal of Physical Oceanography, Vol. 10-8. 1264-1280.

[20] Hasselman et al. (1973). Measurements of Wind-Wave Growth and Swell Decay during the Joint North Sea Project (JONSWAP). Ergansungsheft, Reihe A(8), Nr. 12, Deutschen Hydrografischen Zeitschrift.

[21] Paola, C. Voller, V. R. (2005). A generalized Exner equation for sediment mass balance, J. Geophys. Res., 110.

[22] Yang, C.T. (1995). Sediment transport: theory and practice, Mc Graw Hill Education Europe.

[23] Fowler, A.C. (2001). Dunes and drumlins. Geomorphological fluid mechanics (eds. N.J. Balmforth and A. Provenzale), Springer-Verlag, Berlin, 430454 .

[24] Kouakou K.K.J.; Lagree P-Y, Evolution of a model dune in a shear flow, Eur. J. Mech. B Fluids, 25 no. 3 (2006), pp 348-359. 
[25] Gras, A.J, Sediment transport by waves and currents, SERC London Cent. Mar. Techno, Report No: FL29 (1981).

[26] Kirby, J.T. Dalrymple, R.A. (1995). REFDIF1 v2.5 Refraction diffraction model, Technical report, CACR. Short A.D 1999. Beach and Shoreface Morphodynamics, Wiley.

[27] Hascoet, L. Pascual, V. (2004). Tapenade 2.1 user's guide. INRIA Technical Report RT-300.

[28] Griewank, A. (2001). Computational derivatives, Springer, New York. 